\title{
Mechanistic Insights into the Release of Doxorubicin from Graphene Oxide in Cancer Cells
}

\author{
Erica Quagliarini ${ }^{1, \dagger}$, Riccardo Di Santo ${ }^{2, \dagger}$, Daniela Pozzi ${ }^{2, *}$, Paolo Tentori ${ }^{3,4}$, \\ Francesco Cardarelli ${ }^{4}\left[\right.$ and Giulio Caracciolo ${ }^{2, *}$ \\ 1 Department of Chemistry, Sapienza University of Rome, P.le A. Moro 5, 00185 Rome, Italy; \\ erica.quagliarini@uniroma1.it \\ 2 Department of Molecular Medicine, Sapienza University of Rome, Viale Regina Elena 291, 00161 Rome, Italy; \\ riccardo.disanto@uniroma1.it \\ 3 Center for Nanotechnology Innovation@NEST (CNI@NEST), Istituto Italiano di Tecnologia, Piazza San \\ Silvestro 12, 56127 Pisa, Italy; paolo.tentori@sns.it \\ 4 NEST Laboratory, Scuola Normale Superiore, Piazza San Silvestro 12, 56127 Pisa, Italy; \\ francesco.cardarelli@sns.it \\ * Correspondence: daniela.pozzi@uniroma1.it (D.P.); giulio.caracciolo@uniroma1.it (G.C.) \\ + These authors contributed equally to this work.
}

Received: 10 June 2020; Accepted: 27 July 2020; Published: 29 July 2020

check for updates

\begin{abstract}
Liposomal doxorubicin (L-DOX) is a popular drug formulation for the treatment of several cancer types (e.g., recurrent ovarian cancer, metastatic breast cancer, multiple myeloma, etc.), but poor nuclear internalization has hampered its clinical applicability so far. Therefore, novel drug-delivery nanosystems are actively researched in cancer chemotherapy. Here we demonstrate that DOX-loaded graphene oxide (GO), GO-DOX, exhibits much higher anticancer efficacy as compared to its L-DOX counterpart if administered to cellular models of breast cancer. Then, by a combination of live-cell confocal imaging and fluorescence lifetime imaging microscopy (FLIM), we suggest that GO-DOX may realize its superior performances by inducing massive intracellular DOX release (and its subsequent nuclear accumulation) upon binding to the cell plasma membrane. Reported results lay the foundation for future exploitation of these new adducts as high-performance nanochemotherapeutic agents.
\end{abstract}

Keywords: doxorubicin; functional materials; graphene oxide; nanochemotherapeutics

\section{Introduction}

The anthracycline doxorubicin (DOX) is a common first-line therapy for numerous human pathological conditions such as breast cancer, ovarian cancer, multiple myeloma and Kaposi's sarcoma. In spite of this, DOX recognized adverse side effects, above all cardiotoxicity [1] and nephrotoxicity [2], encouraged the development of alternative nanocarrier-based strategies for safer systemic delivery of DOX. The aim was, in particular, at exploiting the nanocarrier intrinsic ability to protect the drug from degradation, prolong its circulation lifetime, reduce drug dose, finally having a positive impact on drug biodistribution, pharmacokinetics and therapeutic efficacy [3]. In this regard, liposomal DOX (L-DOX) in all its variants (e.g., Doxil ${ }^{\circledR}$, Doxoves ${ }^{\circledR}$ and LipoDox ${ }^{\circledR}$ ) is a paradigmatic example. It has been the first liposomal drug approved by the Food and Drug Administration (FDA) and remains, at present, the drug of choice for the treatment of a number of cancer-related pathologies (mentioned above) [4]. Despite the several advantages conferred by L-Dox formulations (i.e., increased circulation lifetimes as well as enhanced antitumor activity and less toxicity in respect to the free drug), their clinical application is far from being established [5], i.e., almost 10 years after L-DOX-related patents expired, there is still no FDA-approved "generic L-DOX" available. General consensus has 
been reached on the crystalline form of DOX within the lipid vesicles being a major rate-limiting factor for L-DOX efficacy [4]. On one side, it has been suggested that crystalline DOX may favor the rupture of lipid vesicles with the consequent extracellular release of a fraction of the transported payload [6]. On the other side, the largest fraction of internalized crystallized drug stays trapped within liposomes and is shuttled to lysosomes for degradation [7]. Lastly, its interaction with nuclear DNA could be disadvantaged compared to that of its monomeric counterpart.

Based on all these considerations, it is clear that novel nanocarriers that are capable of overcoming the limitations of standard L-DOX formulations are highly desirable in the field. Graphene oxide (GO) is considered a promising nanocarrier due to its peculiar physical-chemical properties such as high surface area, high water dispersibility and good biocompatibility [8]. Compared to other nanocarriers, GO exhibits favorable drug loading capacity due to the two-dimensional planar arrangement of carbon atoms that allows a total exposure of functional groups to the chemical surrounding [9]. Moreover, epoxydic and carboxylic groups on the GO surface can be used to selectively bind drug molecules, thus enhancing drug solubility in aqueous media [10-12]. On the other side, lateral dimensions of GO nanosheets have limitations regarding blood-brain transport, renal clearance, biodegradation and toxicity that have substantially impaired its clinical application so far $[13,14]$.

Here, we explore the potentiality of GO for the efficient loading and intracellular release of DOX in MCF-7 and MDA-MB 231 breast cancer cells [15]. By standard confocal imaging, exploiting DOX intrinsic fluorescence, we demonstrate that GO promotes massive intracellular delivery and subsequent nuclear accumulation of DOX molecules and that such an outcome is followed by high anticancer activity. By phasor-fluorescence lifetime imaging microscopy (FLIM) analysis, in turn, we observe that the DOX molecules within the cytoplasm and nucleus of cells treated with GO-DOX have a characteristic lifetime signature similar to that found in cells treated with the free drug. In addition, GO is detected as attached/adsorbed to the cell plasma membrane, mostly in its unloaded configuration. These observations support the hypothesis of effective drug release from the GO-DOX adduct upon binding of this latter to the cell plasma membrane. Results reported here pave the way to future applications of GO in drug delivery research.

\section{Materials and Methods}

\subsection{Preparation of GO-DOX Complexes}

Graphene oxide (GO) water dispersion was purchased from Graphenea (San Sebastián, Spain). Doxorubicin hydrochloride (DOX) was acquired from Formumax Scientific (Sunnyvale, CA, USA). GO-DOX complexes were prepared by mixing $1 \mathrm{~mL}$ of GO $(0.4 \mathrm{mg} / \mathrm{mL})$ with $1 \mathrm{~mL}$ of DOX water dispersion, at various concentrations. Doing so, we obtained GO-DOX complexes at different GO/DOX weight ratios $\left(R_{W}\right)$, i.e., $R_{W}=3.00,2.00,1,0.50$ and 0.33 . The mixtures were adjusted with $0.01 \mathrm{M} \mathrm{NaOH}$ to have a final $\mathrm{pH}$ value of 8 and sonicated for $3 \mathrm{~min}$ at $125 \mathrm{~W}$ with vibra cell sonicator VC505 (Sonics and Materials, Suffolk, UK). The resulting solutions were left in darkness overnight at $37^{\circ} \mathrm{C}$. Finally, to remove the unbounded DOX, the mixtures were subjected to centrifugation (Hermle Z $216 \mathrm{MK}$, Hermle Labortechnik, Germany) at 18,620 RCF for $30 \mathrm{~min}$ at $4{ }^{\circ} \mathrm{C}$ and the pellet was resuspended with ultrapure water. This procedure was repeated three times. After each washing step, the supernatant was collected and unloaded DOX was quantified by UV-VIS experiments.

\subsection{UV-VIS Experiments}

UV-VIS experiments were performed using a UV-VIS spectrophotometer (JASCO V-570, JASCO Corporation, Tokyo, Japan). As a first step, a DOX calibration curve was generated from a series of DOX solutions with different concentrations (Figure S1 in the Supporting Information). The calibration curve was obtained by plotting the absorbance $480 \mathrm{~nm}$ versus DOX concentration and was treated by linear regression analysis. The linearity of the response of the drug was verified at $1-20 \mu \mathrm{g} / \mathrm{mL}$ 
concentrations. Thus, the DOX loading efficiency (Equation (1)) and loading capacity (Equation (2)) were calculated by the following equations [16]:

$$
\begin{gathered}
\text { Loading Efficiency }(\%)=\frac{\text { Amount of total DOX }- \text { Amount of unloaded DOX }}{\text { Amount of total DOX }} \times 100 \\
\text { Loading Capacity }\left(\frac{\mu \mathrm{g}}{\mu \mathrm{g}}\right)=\frac{\text { Amount of total DOX }- \text { Amount of unloaded DOX }}{\text { Amount of GO }}
\end{gathered}
$$

\subsection{Dynamic Light Scattering}

Size of GO and GO-DOX complexes was determined by dynamic light scattering (DLS). DLS experiments were performed by means of a Zetasizer Nano ZS (Malvern Panalytical, Malvern, UK) at $25{ }^{\circ} \mathrm{C}$. To this end, $100 \mu \mathrm{L}$ of GO or GO-DOX complexes were diluted with $900 \mu \mathrm{L}$ of distilled water (final volume $=1 \mathrm{~mL}$ ). The results are given as mean \pm standard deviation of three independent replicates.

\subsection{Cell Culture}

Breast cancer cell lines (MDA-MB 231 and MCF-7 cells) were purchased from ATCC (Manassas, VA, USA). Cancer cells were preserved in Dulbecco's modified Eagle medium (DMEM) supplemented with $10 \%$ fetal bovine serum (FBS), $1 \%$ penicillin and streptomycin and $1 \%$ of L-glutamine. Cells were maintained at $37^{\circ} \mathrm{C}$ and $5 \% \mathrm{CO}_{2}$ atmosphere.

\subsection{Cell Metabolic Activity Experiments}

For cell metabolic activity experiments, Doxoves ${ }^{\circledR}$ and GO-DOX complexes were administrated to MCF-7 and MDA-MB 231 breast cancer cells. In accordance with previous literature [6,17], two DOX concentrations (13 $\mu \mathrm{g} / \mathrm{well} ; 3 \mu \mathrm{g} /$ well) were tested. Cell metabolic activity of MCF-7 and MDA-MB 231 cells was evaluated by 3-(4,5-dymethyl thiazol 2-y1)-2,5-diphenyl tetrazolium bromide assay (MTT assay, mitochondrial respiration analysis, Sigma-Aldrich, Milan, Italy), according to Mosmann protocol [18]. Water-soluble tetrazolium salt (WST-8) or Cell Counting Kit 8 (MTS) may be used as reliable alternative assays [19]. Briefly, cells were seeded on 96-well plates (10,000 cells/well) for $24 \mathrm{~h}$. After $3 \mathrm{~h}$ incubation in Optimem, the medium was replaced with DMEM 10\% FBS, cells were incubated for $48 \mathrm{~h}$ at $37^{\circ} \mathrm{C}$, and $100 \mu \mathrm{L}$ of isopropyl alcohol was added to each well to dissolve the formazan salt. Finally, the absorbance of each well was obtained with Glomax Discover System (Promega, Madison, WI, USA).

\subsection{Confocal Imaging and Phasor-FLIM Experiments}

Each imaging experiment started $3 \mathrm{~h}$ after the incubation of the cells with GO-DOX and Doxoves ${ }^{\circledR}$, without washing the cells to remove the excess of non-internalized compounds. For imaging, a Leica SP5 confocal microscope (Leica Microsystems AG, Wetzlar, Germany) was used, equipped with an Argon laser for excitation at $488 \mathrm{~nm}$. Doxorubicin emission was collected in the 500-650 nm range. All experiments were performed by using the same imaging settings (e.g., magnification, laser power, etc.). The quantification of the recorded signal was carried out by Image J software (National Institutes of Health, Bethesda, Maryland, USA). During the experiment, the cells were maintained in a thermostatic chamber $\left(37^{\circ} \mathrm{C}, 5 \% \mathrm{CO}_{2}\right)$. For FLIM experiments, all analyzed samples were excited at $470 \mathrm{~nm}$ with a pulsed diode laser operating at $40 \mathrm{MHz}$ (average power: 10-20 $\mu \mathrm{W}$ at the sample) and collecting the emission in the 500-650 $\mathrm{nm}$ range by a photomultiplier tube interfaced with a time-correlated single photon counting card and setup (PicoHarp 300, PicoQuant, Berlin). Phasor analysis of lifetime data was performed by SimFCS software (www.lfd.uci.edu, University of California at Irvine). 


\subsection{Statistical Analysis}

Three replicates of each sample were performed for both cell lines. Values were expressed as average \pm standard deviation. Statistical significance was evaluated using a Student's $t$-test $\left({ }^{*} p<0.05\right.$; ** $p<0.01)$.

\section{Results and Discussion}

Previous studies explored factors affecting DOX loading on GO sheets [20]. Among them, $\mathrm{pH}$ adjustment is considered as a fundamental step for efficient DOX loading by non-covalent bonding. However, controversial results about $\mathrm{pH}$ optimization have been reported. While some studies indicated that DOX loading on GO is maximum at neutral $\mathrm{pH}$ [21], some other identified basic $\mathrm{pH}$ as optimal for loading [12,22]. In a series of preliminary experiments (Figure S2), we found clear evidence that in a basic environment $(\mathrm{pH}=8)$ the complexes show a lower aggregation state respect to other $\mathrm{pH}$ conditions, thus higher DOX loading on GO nanosheets can be achieved. Following $\mathrm{pH}$ adjustment, the role of the GO/DOX weight ratio $\left(R_{W}\right)$ on DOX loading was explored by UV-Vis experiments. To this end, GO-DOX complexes were centrifuged, the pellet was washed three times with ultrapure water and the supernatants carefully collected (Figure 1a).
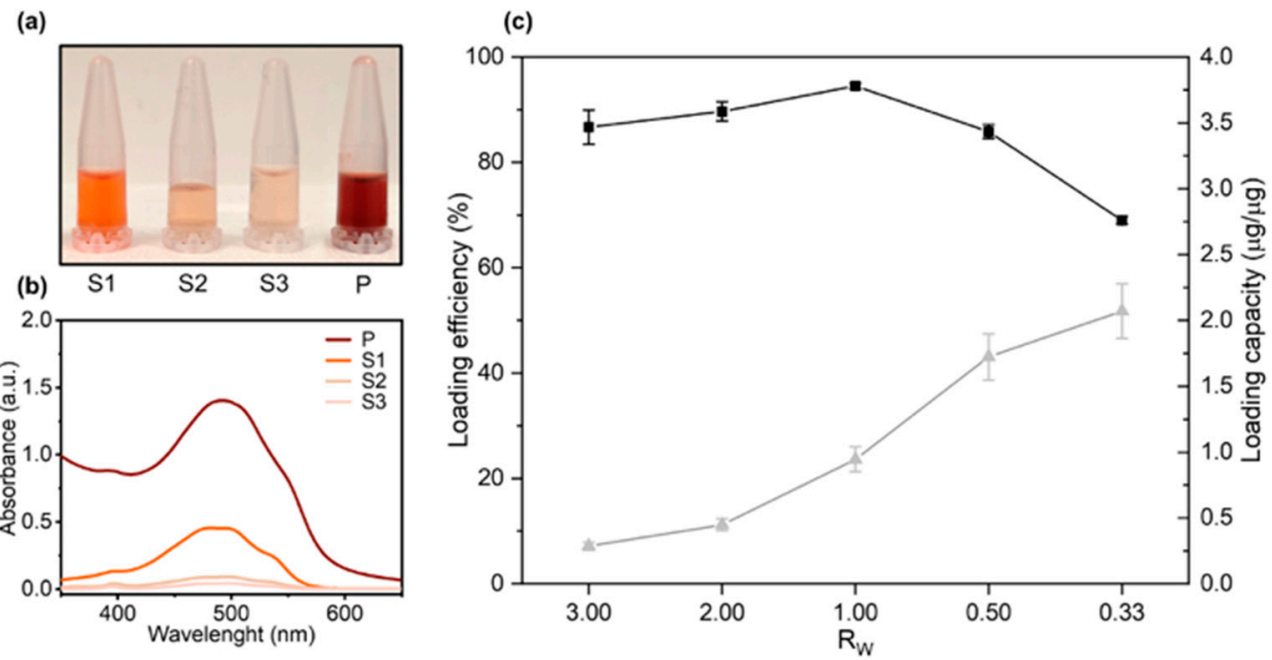

Figure 1. Loading efficiency and loading capacity of doxorubicin (DOX) on graphene oxide (GO) sheets as a function of the GO/DOX weight ratio $R_{W}$. (a) GO-DOX complexes were centrifuged, and the pellet was washed three times with ultrapure water (indicated as S1, S2 and S3 respectively). (b) Absorbance spectra measured from the supernatants. After the third washing step, the pellet was resuspended in ultrapure water and the solution $(\mathrm{P})$ used for (c) quantification of DOX loading efficiency (black squares) and capacity (grey triangles).

Figure $1 \mathrm{~b}$ shows the absorbance spectra measured from wash supernatants in the wavelength range of 350-650 nm. After the third washing step, the absorption peak of DOX was indistinguishable from the background thus ensuring that no significant amount of unbound DOX was present in the sample. The pellet was finally resuspended in ultrapure water (Figure 1a, "P") and the measured peak absorbance (Figure 1b, "P") was used for quantification of DOX loading efficiency and capacity (Figure 1c). As Figure 1c clearly shows, $\mathrm{R}_{\mathrm{W}}=0.33$ allowed one to maximize the DOX loading capacity and was used in the following experiments. The average hydrodynamic size of GO and GO-DOX complexes in aqueous solution was measured by DLS as previously done for large size GO sheets [23]. Data reported in Figure S3 showed that GO-DOX complexes and pristine GO were similar in size with a hydrodynamic diameter (DH) distribution peaked around at $700 \mathrm{~nm}$. Next, GO-DOX complexes were administered to MCF-7 and MDA-MB 231 breast cancer cell lines at two DOX concentrations (13 $\mu \mathrm{g} /$ well and $3 \mu \mathrm{g} /$ well). Pristine GO (i.e., not loaded with DOX) and clinically approved L-DOX in 
the form of Doxoves ${ }^{\circledR}$ were used as controls. Cell metabolic activity was determined using the MTT assay (Figure 2).

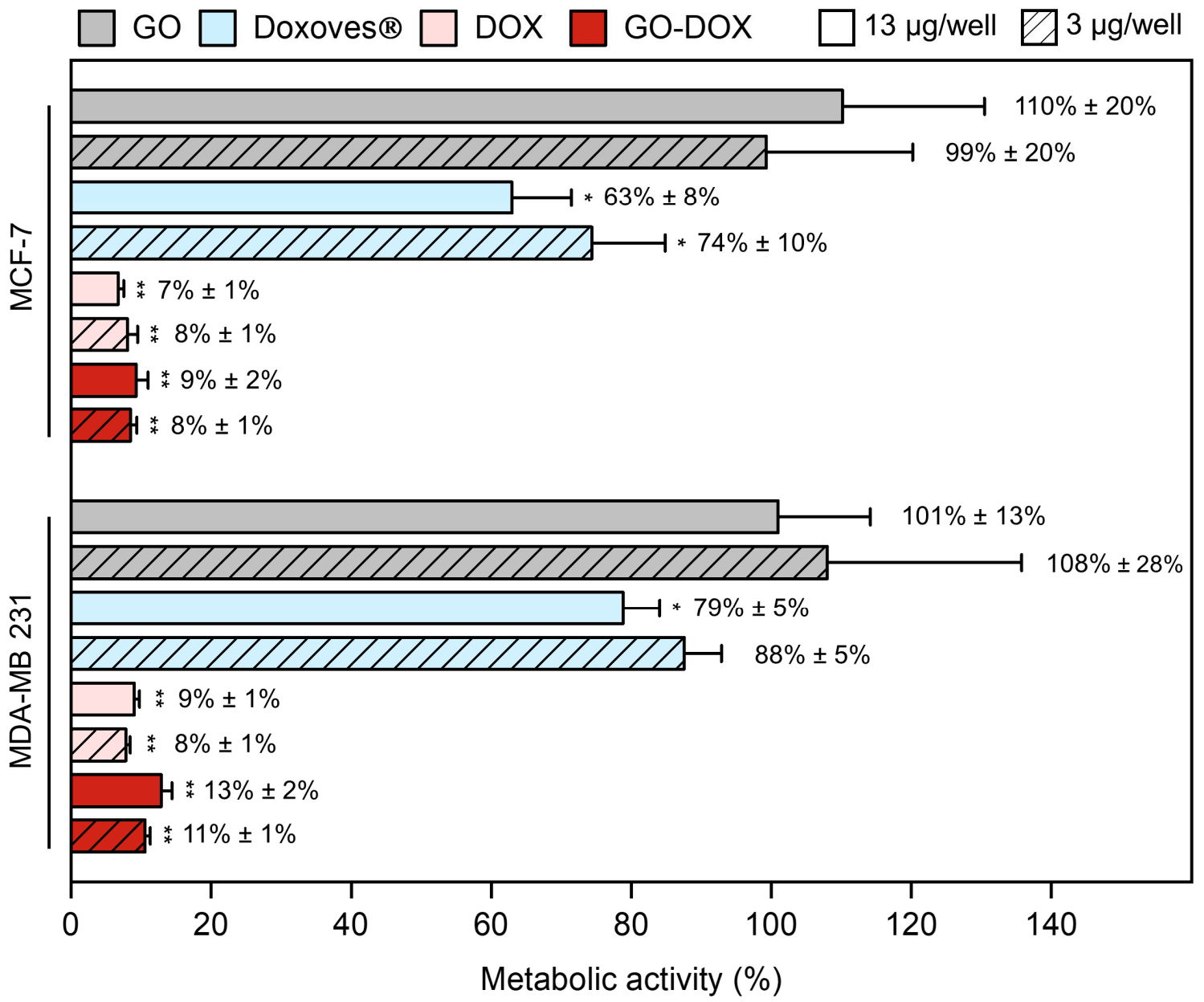

Figure 2. Cell metabolic activity of MCF-7 and MDA-MB 231 cells treated with graphene oxide (GO), commercial liposomal doxorubicin (Doxoves ${ }^{\circledR}$ ), free doxorubicin (DOX) and DOX-loaded GO sheets (GO-DOX) expressed as a percentage with respect to untreated cells. Cell metabolic activity experiments were performed at two different DOX concentrations: i) $13 \mu \mathrm{g} /$ well (empty bars) and $3 \mu \mathrm{g} /$ well (diagonal pattern bars). Results are the average of three replicates \pm standard deviation, normalized. Statistical significance was evaluated with respect to GO using a Student's $t$-test $\left({ }^{*} p<0.05\right.$; $\left.{ }^{* *} p<0.01\right)$.

Cell metabolic activity of MCF-7 and MDA-MB 231 breast cancer cells treated with pristine GO stayed close to $100 \%$ thus confirming that cell death was exclusively due to DNA damage produced by DOX. The administration of free DOX led to a marked reduction of metabolic activity in both cell lines. This result is in line with several previous investigations showing that DOX is one of the most powerful chemotherapeutic agents developed so far. However, as free drugs often undergo premature degradation in the biological environment, encapsulation within nanocarriers is essential to enhance their bioavailability and boost cellular uptake. GO, despite the above-mentioned limitations, is a promising nanoplatform for the delivery of DOX. At high drug concentration, anticancer efficacy of GO-DOX complexes was much higher (residual cell metabolic activity at $9 \%, 24 \mathrm{~h}$ after treatment) than that of commercial Doxoves ${ }^{\circledR}(63 \%)$. Of note, similar results were obtained with MDA-MB 231 cells (cell metabolic activity 13\% and 79\% following treatment with GO-DOX complexes and Doxoves ${ }^{\circledR}$ respectively) and, in both cell lines, at low drug concentration (Figure 2). 
Many anticancer drugs, including DOX, act by intercalating nuclear DNA [24]. Thus, to fully exploit its anticancer efficacy, DOX must be efficiently internalized within cancer cells and subsequently delivered to the cell nucleus. We, therefore, used confocal imaging to explore the intracellular distribution of DOX in cancer cells. Figure 3 (panels a and b) shows representative confocal images of MCF-7 cells treated with Doxoves ${ }^{\circledR}$ and GO-DOX complexes.
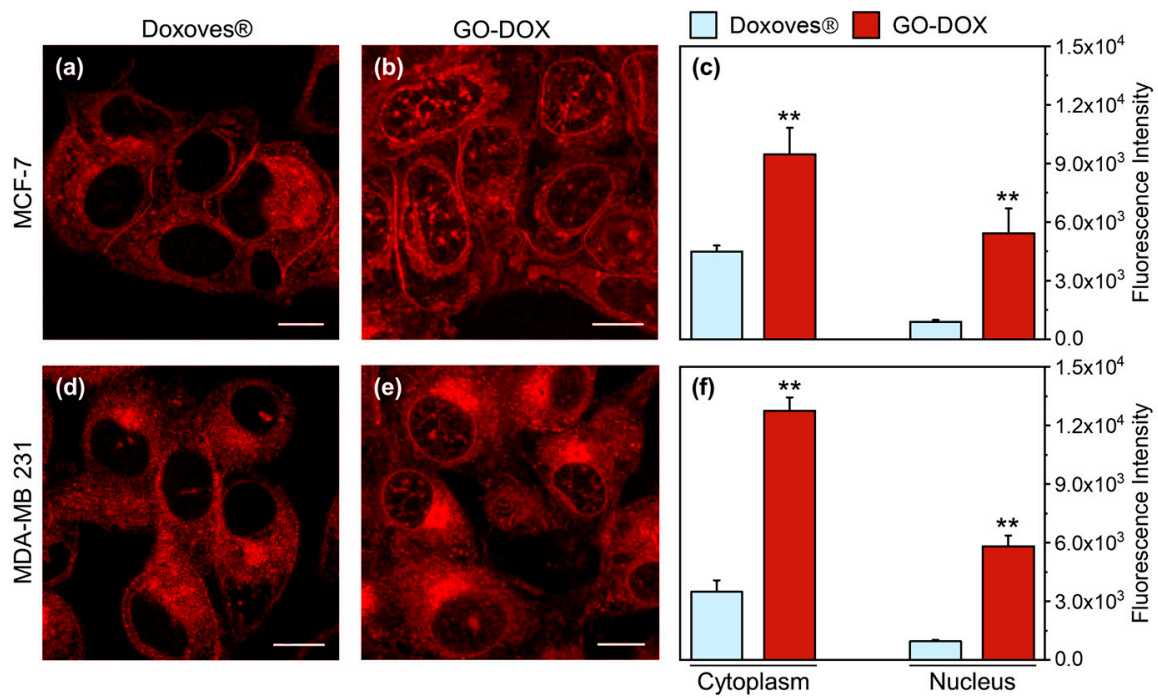

Figure 3. Representative confocal microscopy images of MCF-7 cells treated with (a) Doxoves ${ }^{\circledR}$ and (b) doxorubicin (DOX)-loaded GO sheets GO-DOX complexes (GO/DOX weight ratio, $\mathrm{Rw}=0.33$ ). Experiments were performed at high DOX concentration (i.e., $=13 \mu \mathrm{g} /$ well). (c) Fluorescence intensity analysis of the intracellular localization of DOX. (d-f) Analogously, confocal imaging and analysis were performed in MDA-MB 231 cells. Statistical significance was evaluated using a Student's $t$-test $\left({ }^{* *} p<0.01\right)$ with respect to Doxoves ${ }^{\circledR}$. Calculations reported in panels $\mathrm{c}$ and $\mathrm{f}$ were made using not less than 60 randomly selected cells. Scale bars are $10 \mu \mathrm{m}$.

It is clear, already from visual inspection of images, that GO is able to promote higher cell internalization of DOX as compared to Doxoves ${ }^{\circledR}$. In particular, when MCF-7 cells are treated with GO-DOX, cell nuclei exhibit higher fluorescence signals. By contrast, Doxoves ${ }^{\circledR}$ treatment produces minor, if any, nuclear accumulation of DOX molecules. The histogram plot in Figure 3c reports quantitatively on the nuclear and cytoplasmic signals detected. Worthy of mention, the nuclear fluorescence in cells treated with GO-DOX complexes is five-fold higher as compared to that detected in cells treated with Doxoves ${ }^{\circledR}$. Results were confirmed in MDA-MB 231 cells (Figure 3d-f). Overall, standard confocal imaging suggests that the marked reduction in cell metabolic activity produced by GO-DOX complexes may reflect the higher nuclear content in DOX molecules. Intracellular (and intranuclear) DOX molecules, in turn, are supposed to be free from the GO carrier to explain their functional effect on cell metabolic activity. To support this latter conjecture, we performed fluorescence lifetime imaging microscopy (FLIM) experiments on GO-DOX-treated cells, using the free drug as control (Figure 4). Among the properties of fluorescence, fluorescence lifetime, in fact, can be revelatory of the nanoscale supramolecular organization of the emitter molecule (in this case DOX). In fact, it is expected that DOX in its free form in solution can be distinguished from its counterpart adsorbed/attached to GO or to biological membranes based on the measured characteristic lifetime. The phasor approach to FLIM data is used here as a fit-free, fast and graphical method to extract the quantitative information encrypted in time-domain lifetime measurements [25]. First, we probed the phasor-FLIM signature of free DOX and GO-DOX in solution (Figure S4). Compared to free DOX (mono-exponential lifetime at $1 \mathrm{~ns}$, in keeping with previous reports [26]), GO-DOX shows an average decrease in the characteristic lifetime, which becomes slightly multi-exponential in nature (Figure S4). This latter effect can be ascribed to the contribution of GO intrinsic fluorescence lifetime (measured 
here and found to be $0.1 \mathrm{~ns}$, Figure S4) to the average lifetime of the complex. Worthy of mention, the only minor effect on DOX lifetime induced by its adsorption on GO suggests that that the drug can explore, dynamically, multiple conformations in the complex with GO (in agreement with previous reports [27]).

a

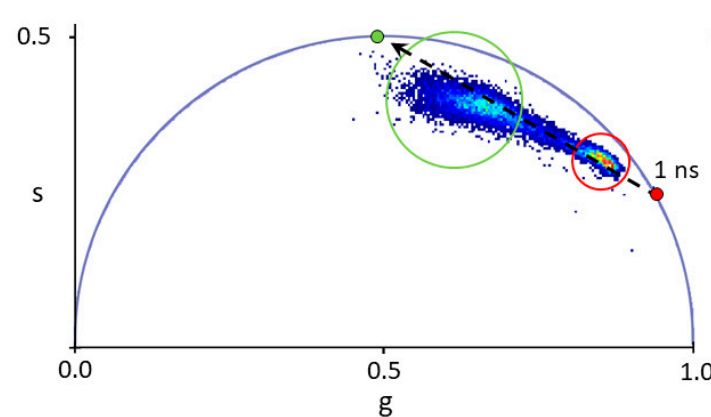

b

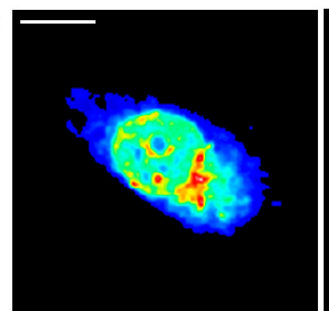

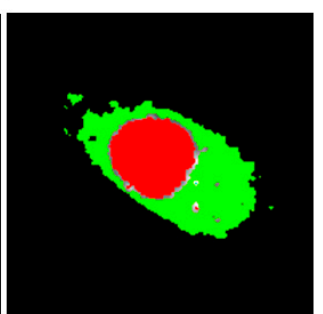

C
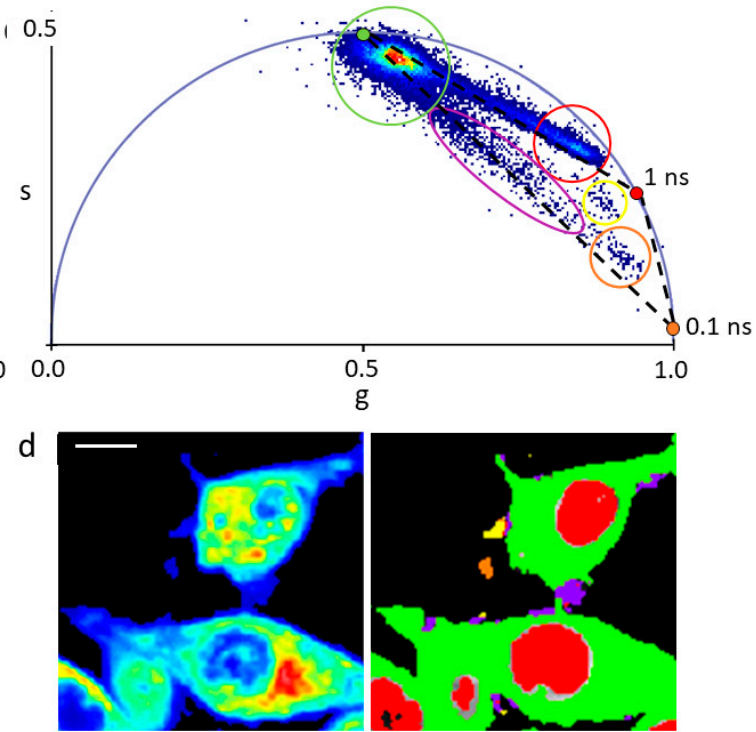

Figure 4. Phasor FLIM analysis of MDA-MB 231 cell exposed to DOX and GO-DOX. (a) Phasor representation of lifetimes measured in cells exposed to DOX. The phasor plot contains clouds of points that correspond to pixels with similar lifetime spectra. These clouds can be selected by specific regions of interest (ROIs). The green ROI, for instance, highlights the portion of pixels corresponding to DOX in the cytoplasm (see right panel in ' $b$ ', the cytoplasm is colored in green); the red ROI clearly identifies pixels corresponding to DOX in the nucleus (see right panel in ' $b$ ', the nucleus is colored in red); a dashed line is drawn across the phasor distribution and used to extrapolate the hypothetical position of a pure species on the universal circle (green point located at $4 \mathrm{~ns}$ ) presumably corresponding to DOX associated to membranes. (b) Intensity (left) and lifetime (right) images of aMDA-MB 231 cell exposed to DOX (the same analyzed in (a)). The lifetime image is colored according to the ROIs in (a). (c,d) Same as before but for MDA-MB 2311 cells exposed to GO-DOX. Please note that the phasor-FLIM signature of cytoplasmic (green cursor in ' $c$ ', green pixels in ' $d$ ') and nuclear DOX signals (red cursor in ' $c$ ', red pixels in ' $d$ ') show clear similarity to that obtained for free DOX. By contrast, as expected, additional signals are present here, which are absent in cells treated with free DOX. In particular, as highlighted by the violet, orange and yellow cursors in ' $c$ ' (and pixels of the same color in ' $\mathrm{d}$ ') there are micrometric patches, mostly associated with cell membranes, with variable amounts of the nude carrier (GO) and the released drug (both free or associated to cellular membranes). Scale bars: $10 \mu \mathrm{m}$.

At this point, we treated MDA-MB 231 cells with DOX and, in parallel, with GO-DOX. The phasor-FLIM analysis is reported in Figure 4.

The fluorescence lifetime spectra measured in each pixel of the image are mapped onto the "phasor" plot: this is made up out of two numbers: the real and imaginary parts (' $g$ ' and 's', amplitude and phase, respectively) of the first harmonic of the Fourier transform of the fluorescence lifetime. Thus, the phasor plot contains clouds of points that correspond to pixels with similar lifetime spectra. These clouds can be selected by specific ROIs, as indicated in Figure 4a,c. As somewhat expected, the characteristic lifetime signature of free DOX transforms into a broad distribution of multi-component lifetimes upon interaction (and mixing) with the complex ensemble of extracellular and intracellular autofluorescent species, which are multi-exponential in nature [28]. In particular, two subclusters are easily recognizable in the phasor plot, one corresponding to DOX molecules localized in the nucleus 
(red circle in Figure 4a, red pixels in Figure 4b), another corresponding to DOX molecules localized in the cytoplasm (green circle in Figure 4a, green pixels in Figure 4b). Please note, also, that the lifetime of DOX in the nucleus is close to that of free DOX in water (indicated by the red point in Figure 4a,c) while, by contrast, DOX lifetime in the cytoplasm shows a sensibly different distribution pattern that can be reasonably ascribed to interaction of DOX with biological membranes (DOX associated to surfaces, in fact, is known to have a lifetime similar to that extrapolated here from data and located at approximately $4 \mathrm{~ns}$ [29], green point in the phasor plot in Figure 4a). What is important for the purpose of this work, however, is that GO-DOX shows some relevant similarities with free DOX in terms of phasor-FLIM signatures within the cell. In particular, the same phasor-plot features described for free DOX are found in cells treated with GO-DOX: an elongated overall distribution with one subcluster corresponding to nuclear DOX (closer to DOX in water, highlighted in red) and another corresponding to cytoplasmic DOX (closer to DOX adsorbed to surfaces/membranes, highlighted in green; Figure 4c). Of note, the lifetime analysis unveils sites of putative GO-DOX attachment to the cell surface (and subsequent drug release), which are not visible in the intensity image (reported in Figure 4d, left panel). As highlighted in Figure 4c, the most important difference in the lifetime data from cells treated with GO-DOX is the presence of pixels with sensibly shorter lifetimes, in particular pointing towards the lifetime of nude GO (i.e., $0.1 \mathrm{~ns}$, orange point). The triangle with vertices the lifetime of the three "pure" species (free DOX, DOX associated to membranes and nude GO) can represent a framework guiding data interpretation. As mentioned above, the phasor data lying along the line between free DOX and membrane-associated DOX recapitulates the main features observed treating cells with free DOX and can be interpreted as an indication that the drug is effectively released at the intracellular level. By contrast, data along the lines connecting nude GO with the other two species unveil the presence of pixels in the image where a variable amount of the carrier (GO) and the released drug (both free or associated to cellular membranes) is detected. We separated this population of pixels into subpopulations of a different color (see violet, orange and yellow cursors in Figure 4c). Of note, however, they all concur to highlight selected micrometric patches on the cell border (Figure $4 \mathrm{~d}$ ). We are prompted to ascribe these patches to sites of GO-DOX cell attachment and subsequent drug release. This observation is in agreement with recent findings obtained using graphene decorated with cyclodextrins and loaded with doxorubicin [30]. The authors, in fact, also due to lifetime analysis, evidenced the efficient cellular uptake of the whole adduct and the presence of DOX in the nucleus without the graphene carrier. Additionally, our data do not contradict previous evidences showing that GO likely binds to integrins at the plasma membrane of cancer cells, activates the integrin-FAK-Rho-ROCK pathway and makes cancer cells more susceptible to chemotherapeutic agents [31]. From a methodological point of view, our results corroborate the idea that FLIM can represent a quantitative platform to analyze DOX cellular uptake and release from nanocarriers, in line with a growing body of evidences from the literature (see for instance References [29,30,32,33]).

\section{Conclusions}

In summary, we have shown that GO can massively deliver free DOX in the nucleus of cancer cells by attaching to the cell membrane and releasing its payload into the cytosol. By this mechanism, GO leads to a significant increase in cell mortality with respect to approved L-DOX. We indeed see this proof of concept work as being part of a future workflow for all bionanoscience, nanomedicine and other areas in which shedding light on the mechanisms of GO-mediated drug delivery is fundamental to fully exploit its potential. The main limitation of the present study is undoubtedly the lateral size of GO-DOX complexes that could be too big for the systemic delivery of DOX [34]. Moreover, in view of in vivo applications, chemical modification of small size GO with hydrophilic polymers [35] such as polyethylene glycol (PEG) or polyethylene oxide (PEO) may also be necessary. Systemic administration of large size GO sheets would likely activate the mononuclear phagocytic system leading to fast removal from the bloodstream. On the other side, other topical medications such as intratumoral and subcutaneous location do not suffer limitations arising from the employment of large size delivery 
systems. Several biomaterials have been used for the local delivery of DOX to breast cancer, skin cancer and colorectal cancer [36,37]. Thus, we suggest that GO sheets used in this work may be useful for these applications. Likewise, clarifying other relevant aspects of the delivery process would be required to strengthen the significance of this study (e.g., apoptosis and invasion/migration) and will be the focus of future investigations. Among other perspectives, coating GO-DOX complexes with a biomolecular corona (i.e., the biomolecular layer surrounding nanomaterials in biological fluids [38-40]) to boost local delivery of DOX could be a promising strategy and will be also addressed in future studies.

Supplementary Materials: The following are available online at http:/www.mdpi.com/2079-4991/10/8/1482/s1, Figure S1: Absorbance spectra of doxorubicin (DOX) at several known concentrations. DOX calibration curve was generated from absorbance values at $\lambda=480 \mathrm{~nm}$, Figure S2: $D_{H}$ of GO/DOX complexes as a function of $\mathrm{pH}$ solution, Figure S3: $\mathrm{D}_{\mathrm{H}}$ intensity-weighted distribution of pristine GO and GO/DOX complexes, Figure S4: The experimental lifetime of DOX in aqueous solution, the experimental lifetime of pristine graphene-oxide (GO) in aqueous solution and the experimental cluster of GO-DOX.

Author Contributions: Conceptualization, F.C., D.P. and G.C.; methodology, E.Q., R.D.S. and P.T.; validation, E.Q., R.D.S. and P.T.; investigation, E.Q., R.D.S. and P.T.; resources, F.C., D.P. and G.C.; data curation, F.C., D.P. and G.C. writing-original draft preparation, E.Q., R.D.S., F.C. and G.C.; writing-review and editing, F.C., D.P. and G.C.; visualization, E.Q., R.D.S. and P.T.; supervision, F.C., D.P. and G.C.; project administration, G.C.; funding acquisition, F.C., D.P. and G.C. All authors have read and agreed to the published version of the manuscript.

Funding: This research received no external funding.

Conflicts of Interest: The authors declare no conflict of interest.

\section{References}

1. Singal, P.K.; Iliskovic, N. Doxorubicin-induced cardiomyopathy. N. Engl. J. Med. 1998, 339, 900-905. [CrossRef] [PubMed]

2. Liu, L.-L.; Li, Q.-X.; Xia, L.; Li, J.; Shao, L. Differential effects of dihydropyridine calcium antagonists on doxorubicin-induced nephrotoxicity in rats. Toxicology 2007, 231, 81-90. [CrossRef]

3. Barenholz, Y.; Cohen, R. Rational design of amphiphile-based drug carriers and sterically stabilized carriers. J. Liposome Res. 1995, 5, 905-932. [CrossRef]

4. Barenholz, Y.C. Doxil ${ }^{\circledR}$ _The first fda-approved nano-drug: Lessons learned. J. Control. Release 2012, 160, 117-134. [CrossRef]

5. Caracciolo, G. Clinically approved liposomal nanomedicines: Lessons learned from the biomolecular corona. Nanoscale 2018, 10, 4167-4172. [CrossRef] [PubMed]

6. Caracciolo, G.; Palchetti, S.; Digiacomo, L.; Chiozzi, R.Z.; Capriotti, A.L.; Amenitsch, H.; Tentori, P.M.; Palmieri, V.; Papi, M.; Cardarelli, F. Human biomolecular corona of liposomal doxorubicin: The overlooked factor in anticancer drug delivery. ACS Appl. Mater. Interfaces 2018, 10, 22951-22962. [CrossRef]

7. Seynhaeve, A.L.; Dicheva, B.M.; Hoving, S.; Koning, G.A.; ten Hagen, T.L. Intact Doxil is taken up intracellularly and released doxorubicin sequesters in the lysosome: Evaluated by in vitro/in vivo live cell imaging. J. Control. Release 2013, 172, 330-340. [CrossRef]

8. Chung, C.; Kim, Y.-K.; Shin, D.; Ryoo, S.-R.; Hong, B.H.; Min, D.-H. Biomedical applications of graphene and graphene oxide. Acc. Chem. Res. 2013, 46, 2211-2224. [CrossRef]

9. Liu, J.; Cui, L.; Losic, D. Graphene and graphene oxide as new nanocarriers for drug delivery applications. Acta Biomater. 2013, 9, 9243-9257. [CrossRef]

10. Liu, Z.; Robinson, J.T.; Sun, X.; Dai, H. PEGylated nanographene oxide for delivery of water-insoluble cancer drugs. J. Am. Chem. Soc. 2008, 130, 10876-10877. [CrossRef]

11. Sun, X.; Liu, Z.; Welsher, K.; Robinson, J.T.; Goodwin, A.; Zaric, S.; Dai, H. Nano-graphene oxide for cellular imaging and drug delivery. Nano Res. 2008, 1, 203-212. [CrossRef] [PubMed]

12. Wu, S.; Zhao, X.; Li, Y.; Du, Q.; Sun, J.; Wang, Y.; Wang, X.; Xia, Y.; Wang, Z.; Xia, L. Adsorption properties of doxorubicin hydrochloride onto graphene oxide: Equilibrium, kinetic and thermodynamic studies. Materials 2013, 6, 2026-2042. [CrossRef] [PubMed]

13. Wu, S.-Y.; An, S.S.A.; Hulme, J. Current applications of graphene oxide in nanomedicine. Int. J. Nanomed. $2015,10,9$. 
14. Shareena, T.P.D.; McShan, D.; Dasmahapatra, A.K.; Tchounwou, P.B. A review on graphene-based nanomaterials in biomedical applications and risks in environment and health. Nano-Micro Lett. 2018, 10, 53. [CrossRef] [PubMed]

15. Lankelma, J.; Dekker, H.; Luque, R.F.; Luykx, S.; Hoekman, K.; Van Der Valk, P.; Van Diest, P.J.; Pinedo, H.M. Doxorubicin gradients in human breast cancer. Clin. Cancer Res. 1999, 5, 1703-1707. [PubMed]

16. Zhao, X.; Liu, L.; Li, X.; Zeng, J.; Jia, X.; Liu, P. Biocompatible graphene oxide nanoparticle-based drug delivery platform for tumor microenvironment-responsive triggered release of doxorubicin. Langmuir 2014, 30, 10419-10429. [CrossRef]

17. Khan, M.S.; Hwang, J.; Lee, K.; Choi, Y.; Seo, Y.; Jeon, H.; Hong, J.W.; Choi, J. Anti-tumor drug-loaded oxygen nanobubbles for the degradation of HIF- $1 \alpha$ and the upregulation of reactive oxygen species in tumor cells. Cancers 2019, 11, 1464. [CrossRef]

18. Mosmann, T. Rapid colorimetric assay for cellular growth and survival: Application to proliferation and cytotoxicity assays. J. Immunol. Methods 1983, 65, 55-63. [CrossRef]

19. Gies, V.; Lopinski, G.; Augustine, J.; Cheung, T.; Kodra, O.; Zou, S. The impact of processing on the cytotoxicity of graphene oxide. Nanoscale Adv. 2019, 1, 817-826. [CrossRef]

20. Hashemi, M.; Yadegari, A.; Yazdanpanah, G.; Omidi, M.; Jabbehdari, S.; Haghiralsadat, F.; Yazdian, F.; Tayebi, L. Normalization of doxorubicin release from graphene oxide: New approach for optimization of effective parameters on drug loading. Biotechnol. Appl. Biochem. 2017, 64, 433-442. [CrossRef]

21. Yang, X.; Zhang, X.; Liu, Z.; Ma, Y.; Huang, Y.; Chen, Y. High-efficiency loading and controlled release of doxorubicin hydrochloride on graphene oxide. J. Phys. Chem. C 2008, 112, 17554-17558. [CrossRef]

22. Zhang, Q.; Li, W.; Kong, T.; Su, R.; Li, N.; Song, Q.; Tang, M.; Liu, L.; Cheng, G. Tailoring the interlayer interaction between doxorubicin-loaded graphene oxide nanosheets by controlling the drug content. Carbon 2013, 51, 164-172. [CrossRef]

23. Choudhary, P.; Parandhaman, T.; Ramalingam, B.; Duraipandy, N.; Kiran, M.S.; Das, S.K. Fabrication of nontoxic reduced graphene oxide protein nanoframework as sustained antimicrobial coating for biomedical application. ACS Appl. Mater. Interfaces 2017, 9, 38255-38269. [CrossRef] [PubMed]

24. Javanbakht, S.; Namazi, H. Doxorubicin loaded carboxymethyl cellulose/graphene quantum dot nanocomposite hydrogel films as a potential anticancer drug delivery system. Mater. Sci. Eng. C 2018, 87, 50-59. [CrossRef]

25. Digman, M.A.; Caiolfa, V.R.; Zamai, M.; Gratton, E. The phasor approach to fluorescence lifetime imaging analysis. Biophys. J. 2008, 94, L14-L16. [CrossRef]

26. Shah, S.; Chandra, A.; Kaur, A.; Sabnis, N.; Lacko, A.; Gryczynski, Z.; Fudala, R.; Gryczynski, I. Fluorescence properties of doxorubicin in PBS buffer and PVA films. J. Photochem. Photobiol. B Biol. 2017, 170, 65-69. [CrossRef]

27. Vovusha, H.; Banerjee, D.; Yadav, M.K.; Perrozzi, F.; Ottaviano, L.; Sanyal, S.; Sanyal, B. Binding characteristics of anticancer drug doxorubicin with two-dimensional graphene and graphene oxide: Insights from density functional theory calculations and fluorescence spectroscopy. J. Phys. Chem. C 2018, 122, 21031-21038. [CrossRef]

28. Stringari, C.; Cinquin, A.; Cinquin, O.; Digman, M.A.; Donovan, P.J.; Gratton, E. Phasor approach to fluorescence lifetime microscopy distinguishes different metabolic states of germ cells in a live tissue. Proc. Natl. Acad. Sci. USA 2011, 108, 13582-13587. [CrossRef]

29. Basuki, J.S.; Duong, H.T.; Macmillan, A.; Erlich, R.B.; Esser, L.; Akerfeldt, M.C.; Whan, R.M.; Kavallaris, M.; Boyer, C.; Davis, T.P. Using fluorescence lifetime imaging microscopy to monitor theranostic nanoparticle uptake and intracellular doxorubicin release. ACS Nano 2013, 7, 10175-10189. [CrossRef]

30. Caccamo, D.; Currò, M.; Ientile, R.; Verderio, E.A.; Scala, A.; Mazzaglia, A.; Pennisi, R.; Musarra-Pizzo, M.; Zagami, R.; Neri, G. Intracellular fate and impact on gene expression of doxorubicin/cyclodextrin-graphene nanomaterials at sub-toxic concentration. Int. J. Mol. Sci. 2020, 21, 4891. [CrossRef]

31. Zhu, J.; Xu, M.; Gao, M.; Zhang, Z.; Xu, Y.; Xia, T.; Liu, S. Graphene oxide induced perturbation to plasma membrane and cytoskeletal meshwork sensitize cancer cells to chemotherapeutic agents. ACS Nano 2017, 11, 2637-2651. [CrossRef]

32. Zhang, X.; Shastry, S.; Bradforth, S.E.; Nadeau, J.L. Nuclear uptake of ultrasmall gold-doxorubicin conjugates imaged by fluorescence lifetime imaging microscopy (FLIM) and electron microscopy. Nanoscale 2015, 7, 240-251. [CrossRef] [PubMed] 
33. Suarasan, S.; Craciun, A.-M.; Licarete, E.; Focsan, M.; Magyari, K.; Astilean, S. Intracellular dynamic disentangling of doxorubicin release from luminescent nanogold carriers by fluorescence lifetime imaging microscopy (FLIM) under two-photon excitation. ACS Appl. Mater. Interfaces 2019, 11, 7812-7822. [CrossRef] [PubMed]

34. Barua, S.; Mitragotri, S. Challenges associated with penetration of nanoparticles across cell and tissue barriers: A review of current status and future prospects. Nano Today 2014, 9, 223-243. [CrossRef] [PubMed]

35. Wang, L.; Yu, D.; Dai, R.; Fu, D.; Li, W.; Guo, Z.; Cui, C.; Xu, J.; Shen, S.; Ma, K. PEGylated doxorubicin cloaked nano-graphene oxide for dual-responsive photochemical therapy. Int. J. Pharm. 2019, 557, 66-73. [CrossRef]

36. Huber, L.A.; Pereira, T.A.; Ramos, D.N.; Rezende, L.C.; Emery, F.S.; Sobral, L.M.; Leopoldino, A.M.; Lopez, R.F. Topical skin cancer therapy using doxorubicin-loaded cationic lipid nanoparticles and iontophoresis. J. Biomed. Nanotechnol. 2015, 11, 1975-1988. [CrossRef]

37. Zhao, N.; Woodle, M.C.; Mixson, A.J. Advances in delivery systems for doxorubicin. J. Nanomed. Nanotechnol. 2018, 9, 519. [CrossRef]

38. Capriotti, A.L.; Caracciolo, G.; Caruso, G.; Cavaliere, C.; Pozzi, D.; Samperi, R.; Laganà, A. Analysis of plasma protein adsorption onto DC-Chol-DOPE cationic liposomes by HPLC-CHIP coupled to a Q-TOF mass spectrometer. Anal. Bioanal. Chem. 2010, 398, 2895-2903. [CrossRef]

39. Palchetti, S.; Pozzi, D.; Capriotti, A.L.; La Barbera, G.; Chiozzi, R.Z.; Digiacomo, L.; Peruzzi, G.; Caracciolo, G.; Laganà, A. Influence of dynamic flow environment on nanoparticle-protein corona: From protein patterns to uptake in cancer cells. Colloids Surf. B Biointerfaces 2017, 153, 263-271. [CrossRef]

40. Caracciolo, G.; Caputo, D.; Pozzi, D.; Colapicchioni, V.; Coppola, R. Size and charge of nanoparticles following incubation with human plasma of healthy and pancreatic cancer patients. Colloids Surf. B Biointerfaces 2014, 123, 673-678. [CrossRef] [PubMed]

(C) 2020 by the authors. Licensee MDPI, Basel, Switzerland. This article is an open access article distributed under the terms and conditions of the Creative Commons Attribution (CC BY) license (http://creativecommons.org/licenses/by/4.0/). 